\title{
Chromosome-Wide Characterization of Intragenic Crossover in Shiitake Mushroom, Lentinula edodes
}

\author{
Wenbing Gong ${ }^{1,2}$, Nan Shen ${ }^{1}$, Lin Zhang ${ }^{1}$, Yinbing Bian ${ }^{1}$ and Yang Xiao ${ }^{1, *(1)}$ \\ 1 Institute of Applied Mycology, Huazhong Agricultural University, Wuhan 430070, China; \\ gongwenbing@caas.cn (W.G.); bioshennan@foxmail.com (N.S.); cai284829597@126.com (L.Z.); \\ bianyinbing@mail.hzau.edu.cn (Y.B.) \\ 2 Institute of Bast Fiber Crops, Chinese Academy of Agricultural Sciences, Changsha 410205, China \\ * Correspondence: xiaoyang@mail.hzau.edu.cn or xyfungi@163.com
}

Citation: Gong, W.; Shen, N.;

Zhang, L.; Bian, Y.; Xiao, Y.

Chromosome-Wide Characterization of Intragenic Crossover in Shiitake Mushroom, Lentinula edodes. J. Fungi 2021, 7, 1076. https://doi.org/ $10.3390 /$ jof7121076

Academic Editor: Willem Melchers

Received: 4 November 2021

Accepted: 6 December 2021

Published: 15 December 2021

Publisher's Note: MDPI stays neutral with regard to jurisdictional claims in published maps and institutional affiliations.

Copyright: (c) 2021 by the authors. Licensee MDPI, Basel, Switzerland. This article is an open access article distributed under the terms and conditions of the Creative Commons Attribution (CC BY) license (https:// creativecommons.org/licenses/by/ $4.0 /)$.

\begin{abstract}
Meiotic crossover plays a critical role in generating genetic variations and is a central component of breeding. However, our understanding of crossover in mushroom-forming fungi is limited. Here, in Lentinula edodes, we characterized the chromosome-wide intragenic crossovers, by utilizing the single-nucleotide polymorphisms (SNPs) datasets of an $\mathrm{F}_{1}$ haploid progeny. A total of 884 intragenic crossovers were identified in 110 single-spore isolates, the majority of which were closer to transcript start sites. About $71.5 \%$ of the intragenic crossovers were clustered into 65 crossover hotspots. A 10 bp motif (GCTCTCGAAA) was significantly enriched in the hotspot regions. Crossover frequencies around mating-type A (MAT-A) loci were enhanced and formed a hotspot in L. edodes. Genome-wide quantitative trait loci (QTLs) mapping identified sixteen crossoverQTLs, contributing 8.5-29.1\% of variations. Most of the detected crossover-QTLs were co-located with crossover hotspots. Both cis- and trans-QTLs contributed to the nonuniformity of crossover along chromosomes. On chr2, we identified a QTL hotspot that regulated local, global crossover variation and crossover hotspot in L. edodes. These findings and observations provide a comprehensive view of the crossover landscape in L. edodes, and advance our understandings of conservation and diversity of meiotic recombination in mushroom-forming fungi.
\end{abstract}

Keywords: Lentinula edodes; intragenic crossover; hotspots; cis- and trans-loci

\section{Introduction}

Meiotic recombination is an essential cellular process shared across eukaryotes, which recombines genetic variation and plays a pivotal role in genome evolution [1,2]. During meiosis, DNA double-strand breaks (DSBs) enter interhomolog repair to yield two types of recombination products, crossovers and non-crossovers [2,3]. Meiotic crossovers between homologous chromosomes break-up haplotypes and shuffle genetic variations, and are essential for variety breeding, which relies on the ability to combine favorable alleles [4]. Thereby, the profound understanding of molecular mechanisms driving meiotic crossovers has the potential to accelerate variety breeding and allow the more effective use of the available variation [5].

The occurrence of meiotic crossovers along chromosomes is highly non-random. Crossovers are preferentially located in kilobase-sized regions known as crossover hotspots [6]. During the past decades, the meiotic recombination process has been extensively studied in model species and has provided important insights into conserved mechanisms [6,7]. Meanwhile, meiotic crossovers vary considerably between species, populations and individuals, and the regulations of crossover variation are under very complicated cis- and trans-genetic control [8-10]. Unique and novel meiotic crossover features have also been observed in mushroomforming basidiomycetes. For instance, in a hypervariable basidiomycete Schizophyllum commune, meiotic crossover preferentially occurred in regions of high local similarity [11]. Extremely, in a homothallic basidiomycete Agaricus bisporus, meiotic crossovers were almost restricted 
exclusively to regions of about $100 \mathrm{~kb}$ at the chromosome ends [12]. The extensive investigation of meiotic crossover in mushroom-forming basidiomycete may help to shape our understanding of conservation and diversity in meiotic strategies.

Lentinula edodes, also called the shiitake mushroom, is renowned for its compelling health-promoting properties [13]. Currently, L. edodes is widely cultivated and it contributes to about $22 \%$ of the world's total mushroom production [14]. Given its importance in research and the food industry, L. edodes has been extensively studied as a model basidiomycete for mushroom genetics and physiology research. Recently, we re-assembled a chromosome-level genome and genotyped an $\mathrm{F}_{1}$ haploid progeny by high-throughput RNA-seq [15]. These $F_{1}$ haploid progenies were derived from products of one generation of meiosis and were therefore suitable to dissect meiotic crossover events. Herein, we re-mapped the single-nucleotide polymorphisms (SNPs) into the linkage map of L. edodes, and identified genome-wide intragenic crossovers by haplotype switches along the chromosomes. The crossover hotspots and genetic architecture of crossover variation in these $F_{1}$ haploid progenies were also characterized. Findings in this study may advance our understandings of meiotic recombination in mushroom-forming basidiomycetes.

\section{Materials and Methods}

\subsection{Haploid Progeny Population}

The $\mathrm{F}_{1}$ haploid progenies, comprising 110 single-spore isolates (SSIs) of the hybrid strain L205-6 × W1-26, were generated and utilized previously [15,16]. Genomes of the two parent monokaryons L205-6 and W1-26 had been re-sequenced [17]. Recently, via highthroughput RNA-seq and genotypes deduction, we genotyped the 110 SSIs and constructed a high-density genetic map, Lemap2.0, comprising 488 recombination bins [15]. A $37.23 \mathrm{Mb}$ genome sequence of L. edodes was also re-anchored into nine pseudo-chromosomes (https: //mycocosm.jgi.doe.gov/Lenedo1, accessed on: 12 May 2021), which aligned into nine linkage groups of Lemap2.0 [15]. All the sequencing data are deposited into GenBank under the accession of PRJNA649389 (https:/ / www.ncbi.nlm.nih.gov/bioproject/PRJNA649389, accessed on: 22 November 2021).

\subsection{Identification of Crossovers and Hotspots}

In order to identify crossovers, we re-mapped all 69,634 markers (SNPs and InDels) into Lemap2.0 [15]. According to the recombination studies in plants $[9,18]$, crossover events (recombination breakpoints) were identified by the changes in the parental genotype phase ordered along the chromosomes. A python script "findCrossover.py" (Supplementary File S1) was written to count the number of crossover events per chromosome for each individual. To reduce the influence of mis-genotyped and misplaced markers on identification of crossovers, one crossover event was recorded in a case where at least five consecutive markers on both sides of the recombination breakpoint were from different parents [19]. Meiotic crossovers do not occur uniformly along the chromosomes. In this study, we divided the L. edodes genome into non-overlapping $10 \mathrm{~kb}$ windows and recorded crossovers in each window. To identify crossover hotspots, the Poisson function in Excel was used to find the threshold value of the number of crossovers in each $10 \mathrm{~kb}$ window $[20,21]$. Then, the motif enrichment analysis in crossover hotspots was performed using the Homer findMotifsGenome module in Homer software v4.10.1 [22].

\subsection{Quantitative Trait Loci (QTLs) Mapping of Crossover Variation}

In order to detect genetic factors conferring crossover variation in L. edodes, total number of crossover events (TCO), crossover events in hotspots (HCO) and crossover events of each chromosome (COchr) were used as the phenotype dataset for QTL analysis. Combining Lemap2.0, genotypic data and phenotypic data of the 110 SSIs, QTLs underlying crossover variation were mapped using composite interval mapping with WinQTLCart 2.5 [23]. Parameters for QTL detection were set to model 6 with a scanning step of $1 \mathrm{cM}$ (number of control markers $=5$; window size $=10 \mathrm{cM}$ ). The 1000-permutation-based LOD 
threshold was calculated to declare the presence of a QTL $(p<0.05)$. Confidence intervals (CIs) of QTLs were supported by LOD peak minus one. Detailed descriptions (position, additive effect, LOD score, $\mathrm{CI}$ and percentage of phenotypic variation explained) were provided to depict each QTL [17]. For the QTL mapping of crossover variation on each chromosome, if QTL was located in the chromosome, of which the crossover number was analyzed, such QTL was defined as cis-prone crossover QTL. If QTL is not mapped on the chromosome, of which the crossover number was analyzed, the QTL was defined as trans-crossover QTL. Genes in the CIs of QTLs were analyzed to screen for candidate genes regulating crossover variation.

\section{Results and Discussion}

\subsection{Distribution of Crossovers in Haploid Progenies}

In previous work, crossover events were extensively quantified by re-sequencing the recombined gametes derived from meiosis, such as in human [24], yeast [25], Arabidopsis [26] and also in fungi [11]. Here, the $110 \mathrm{~F}_{1}$ SSIs of L. edodes were the recombined basidiospores derived from meiosis. Therefore, the crossover events could be scored by counting the recombination breakpoints, in which change of linkage phase between markers indicates the occurrence of a crossover [5]. Moreover, to avoid false crossover detection, a rigorous criterion for haplotype switches was employed. We define a crossover as a change in parental genotype phase for at least five consecutive markers [19].

The feature of crossover distribution in L. edodes was summarized in Supplementary Table S1. In the $110 \mathrm{~F}_{1}$ meiotic SSIs, a total of 884 intragenic crossovers (TCOs) were identified. Results of the number of crossovers suggested that limited crossovers occurred per chromosome per individual, varying from 0.25 to 1.80 (Supplementary Table S1). The low frequency of crossovers per chromosome might be due to crossover interference, i.e., crossover in one genetic interval reducing the likelihood of a co-occurring crossover in an adjacent interval [6,27]. Here, in L. edodes, there was a sizeable proportion of progenies that did not show crossover, which was in line with Zymoseptoria tritici [28]. The occurrence of achiasmatic chromosomes was also observed in mushroom-forming basidiomycetes, such as Hericium erinaceus [19] and Pleurotus ostreatus [29]. Overall, the low number of crossovers may limit the efficiency of the breeding process [4].

The number of crossovers per SSI ranged from 4 to 16, showing continuous distribution, and suggesting their nature of quantitative traits (Supplementary Figure S1). Comparatively, the number of crossovers per individual in L. edodes (average 8.04) was on the same order of magnitude as other heterothallic basidiomycetes, such as 10.4 in H. erinaceus [19] and 4.2 in S. commune [11], but larger than the homothallic basidiomycete A. bisporus, in which only an average of 0.5 crossovers per individual was observed [12]. Similar to H. erinaceus [19], the number of crossovers was related to the chromosome length, and long chromosomes tended to have a higher number of average crossovers (Supplementary Table S2).

Among these 884 intragenic crossovers, the majority of intragenic crossovers (59.7\%) were closer to transcript start sites, which was consistent with the pattern observed in yeast [30] and maize [8], but differs from recombination studies in humans, where crossovers decreased towards gene transcripts [31]. This may indicate that in L. edodes, recombination-driven genomic exchanges tend to alter the promoter or regulatory regions of genes.

\subsection{Crossover Hotspots and Mating Type}

In most eukaryotes, crossovers are non-uniformly distributed along chromosomes and typically cluster within hotspots [32]. It has been widely reported in fungi that crossovers preferentially located near telomeres and sub-telomeric regions $[12,19,28,29]$. Here, in L. edodes, we recorded crossovers in non-overlapping $10 \mathrm{~kb}$ segments and identified hotspot regions with large numbers of crossovers (Figure 1). In these $\mathrm{F}_{1}$ haploid progenies, an average of 0.23 crossovers were recorded per $10 \mathrm{~kb}$ segments of the L. edodes genome. 
The regions with at least four crossovers per $10 \mathrm{~kb}$ segment were identified as crossover hotspots (Poisson distribution test, $p<0.0001$ ). Thereby, we found 65 crossover hotspots in the L. edodes genome, where the crossovers reached to 17-117 times the genome average (Supplementary Table S3). These hotspots were scattered onto eight chromosomes, but not on chr7. Although the underlying genetic mechanism was unknown, the whole chr7 seemed to be recombination-suppressed (Figure 1). Recombination suppression of whole chromosomes was also clearly shown in A. bisporus [12,33]. The longest chromosome, chr1, had the most crossover hotspots ( 15 of 65 ; Supplementary Table S3). However, the largest number of crossovers was observed on the $10 \mathrm{~kb}$ hotspot region on chr3 (920-930 kb), which was 117-fold higher than the genomic background (Supplementary Table S3). These 65 crossover hotspots represented $1.7 \%$ of the genome, and contained $71.5 \%$ (632 of 884 ) of the intragenic crossovers. These 632 crossovers in hotspots were therefore considered as HCOs. HCO was significantly positively correlated with TCO $\left(\mathrm{r}=0.745, p<1.2 \times 10^{-20}\right)$. SNP frequency in crossover hotspots was higher than that of genome average (ANOVA, $p<0.006)$. In crossover hotspots, enrichment analysis also revealed a significantly enriched motif (GCTCTCGAAA) similar to the binding motif of SWI4, a transcription factor that is an essential part of a complex involved in cell-cycle-dependent transcription in yeast [34] $\left(p<1 \times 10^{-8}\right)$. The existence of crossover hotspots was in agreement with observations in other fungi: $1.4 \%$ of most active sites correspond to $32.3 \%$ of crossover in H. erinaceus [19] and $1.9-3.3 \%$ of the genome contained $27.8-38.2 \%$ of crossovers in Z. tritici [28]. Additionally, in yeast, $10 \%$ of the most active sites corresponded to more than $50 \%$ of all recombination events [30]. The distribution of crossovers in L. edodes seems to be more concentrated, which might be because they were intragenic crossovers.

Many sexually reproducing species distribute crossovers in a sex-specific pattern along chromosomes [35-37]. In fungi, recombination also plays critical roles in matingtype determination [38]. Here, in L. edodes, we tested the effect of mating type of SSIs on TCO and HCO. Weak differences of TCO and HCO were observed among the SSIs with different mating-type A (MAT-A) loci. The average TCO and HCO of the SSIs with MAT-A $_{1}$ (same as the parent monokaryon L205-6) were slightly higher than those of MAT- $A_{2}$ SSIs (same as W1-26) (Supplementary Figure S2). This result differed from that of $H$. erinaceus, in which no significant difference was observed among total crossovers of different mating-type SSIs [19]. Differences in viability, germination, or vegetative growth rate associated with different mating haplotypes may lead to selection bias of monokaryons [39], and further lead to crossover bias. Here, in the 110 SSIs of L. edodes, no distorted segregation was observed for MAT-A loci (MAT-A A $_{1}$-SSIs : MAT-A A $_{2}$-SSIs was $53: 57)$. There seems to be other reasons for crossover bias between mating types. In animals and plants, extensive recombination differences between sexes are observed [36,37]. In basidiomycetes, whether and why there is crossover bias between mating types needs extensive research. Interestingly, the region surrounding the MAT-A loci formed a crossover hotspot (Hotspot20) in L. edodes (Supplementary Table S3), while that region was characterized by suppressed recombination in H. erinaceus [19]. In fact, although the evolutionary drivers are still unclear, contrasting relationships between recombination and mating-type determination are observed $[38,40]$. Regions involved in mating compatibility are in some cases associated with recombination suppression, while in other cases with enhanced recombination [40].

\subsection{Regulation of Meiotic Crossovers}

The regulation of meiotic crossovers is very complex and multilevel, including genome-wide mechanisms, chromosome-specific phenomena and local effects [6]. In recent years, great efforts have been made to increase the insight into recombination in model species, and several loci and genes that shape crossover variation have been identified [9]. Additionally, the QTL mapping method was employed to dissect the genetic mechanisms underlying crossover variation in animals and plants $[9,18,41,42]$. Here, in L. edodes, to decipher the genetic factors conferring the non-uniformity of crossovers, TCO, 
$\mathrm{HCO}$ and COchr were taken as phenotypic traits to perform QTL mapping. TCO indicated global crossover events across the genome, $\mathrm{HCO}$ evaluated crossovers in specific hotspot regions, while COchr would be more accurate to quantify crossovers for each chromosome.
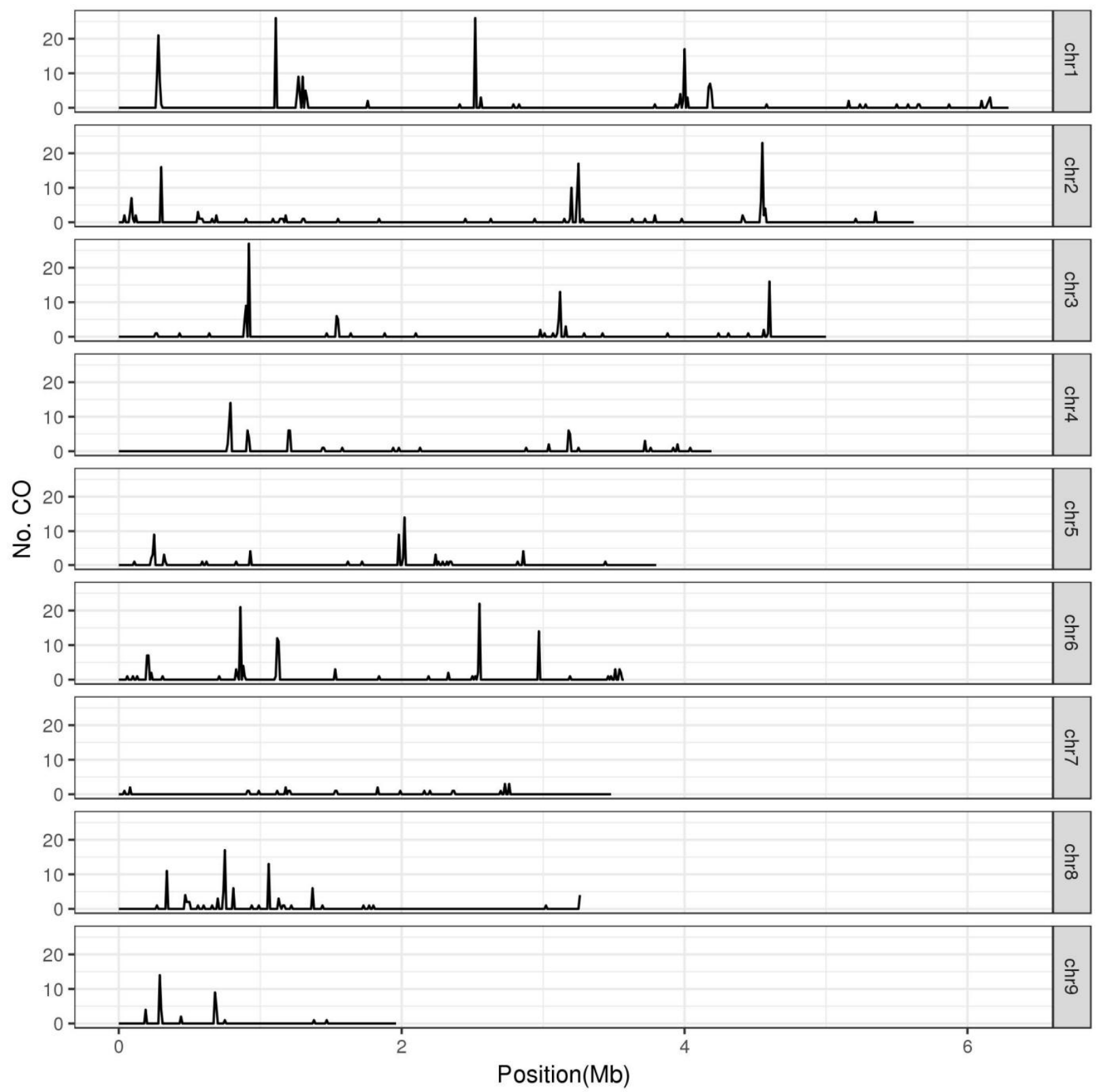

Figure 1. Distribution of crossover events along the chromosomes of L. edodes. The L. edodes genome was divided into non-overlapping $10 \mathrm{~kb}$ windows and recorded the crossover events of each window. The regions, for which no less than four crossovers per $10 \mathrm{~kb}$ segment were identified as crossover hotspots.

In total, sixteen loci, including one TCO-QTL, two HCO-QTLs and thirteen COchrQTLs, were mapped for crossover variation (Table 1, Figure 2). Similar to H. erinaceus [19], most of the detected crossover-QTLs were within or next to the crossover hotspots, suggesting conservation of the mechanism in basidiomycetes, i.e., genes in hotspots shaped crossover variation in hotspots, and further regulated the local and global crossover. The locus tco. 1 was mapped on chr2, contributing $10.6 \%$ of global crossover variation across the genome. For crossover events in hotspots, $h c o .1$ and $h c o .2$ accounted for $20.9 \%$ of HCO variation. The tco.1 and $h c o .2$, both showing negative additive effects, were close together on chr2, which suggests the existence of linked genes regulating TCO and HCO. The linkage between QTLs for TCO and HCO contributed to their phenotypic correlation. 
Table 1. QTLs for crossover variation of L. edodes.

\begin{tabular}{|c|c|c|c|c|c|c|c|c|c|c|c|}
\hline Locus & Trait & Chr & Type & Position (cM) & LOD & Additive & $R^{2}(\%)$ & ${ }^{\text {a }} \mathrm{CI}$ (cM) & $\begin{array}{l}\text { b No. of } \\
\text { Genes }\end{array}$ & $\begin{array}{l}\text { c Association } \\
\text { with Hotspots }\end{array}$ & $\begin{array}{l}\text { Length of } \\
\text { CI (kb) }\end{array}$ \\
\hline cochr1.1 & COchr1 & 1 & $\begin{array}{l}\text { cis- } \\
\text { prone }\end{array}$ & 83.7 & 3.07 & 0.61 & 11.0 & $81.9-90.2$ & 6 & hotspot8 & 29.4 \\
\hline $\operatorname{coch} r 2.1$ & COchr2 & 1 & trans & 155.5 & 2.95 & -0.33 & 8.5 & $\begin{array}{l}152.5- \\
158.2\end{array}$ & 5 & hotspot13 & 9.5 \\
\hline $\operatorname{coch} r 2.2$ & COchr2 & 1 & trans & 162.8 & 3.81 & -0.41 & 10.8 & $\begin{array}{l}160.6- \\
164.3\end{array}$ & 2 & hotspot14 & 2.0 \\
\hline cochr 2.3 & COchr2 & 8 & trans & 1.9 & 3.64 & 0.31 & 10.6 & $0-6.8$ & 46 & hotspot54 & 203.0 \\
\hline $\operatorname{coch} r 3.1$ & COchr3 & 2 & trans & 53.4 & 2.88 & 0.46 & 9.5 & $50.5-56.2$ & 2 & $\begin{array}{c}\text { near to } \\
\text { hotspot18 }\end{array}$ & 5.2 \\
\hline cochr 3.2 & COchr3 & 2 & trans & 80.0 & 3.33 & -0.33 & 11.0 & $74.7-81.0$ & 5 & hotspot 20 & 5.6 \\
\hline cochr3.3 & COchr3 & 2 & trans & 85.5 & 3.04 & -0.30 & 10.0 & $81.9-87.6$ & 290 & $\begin{array}{c}\text { near to } \\
\text { hotspot20 }\end{array}$ & 721.9 \\
\hline cochr4.1 & COchr4 & 4 & $\begin{array}{l}\text { cis- } \\
\text { prone }\end{array}$ & 45.0 & 8.32 & -0.96 & 29.1 & $44.5-45.1$ & 112 & / & 396.4 \\
\hline $\operatorname{cochr} 4.2$ & COchr4 & 4 & $\begin{array}{l}\text { cis- } \\
\text { prone }\end{array}$ & 56.0 & 8.46 & 0.85 & 24.1 & $55.0-58.0$ & 3 & hotspot38 & 6.2 \\
\hline cochr6.1 & COchr6 & 6 & $\begin{array}{l}\text { cis- } \\
\text { prone }\end{array}$ & 10.1 & 4.59 & -0.72 & 15.1 & 9.9-14.4 & 4 & hotspot46 & 23.5 \\
\hline cochr7.1 & COchr7 & 7 & $\begin{array}{l}\text { cis- } \\
\text { prone }\end{array}$ & 2.8 & 5.82 & -0.25 & 21.4 & $1.6-4.1$ & 247 & / & 836.5 \\
\hline cochr8.1 & COchr8 & 1 & trans & 97.3 & 3.35 & -0.34 & 9.3 & $94.2-99.9$ & 44 & $\begin{array}{c}\text { near to } \\
\text { hotspot10 }\end{array}$ & 117.3 \\
\hline cochr 8.2 & COchr8 & 1 & trans & 105.5 & 3.40 & -0.34 & 8.7 & $\begin{array}{l}103.6- \\
113.2\end{array}$ & 11 & hotspot10 & 37.5 \\
\hline hco.1 & $\begin{array}{l}\mathrm{HCO} \\
\mathrm{HCO}\end{array}$ & 1 & ' & $\begin{array}{c}5.8 \\
756\end{array}$ & $\begin{array}{l}3.26 \\
3.73\end{array}$ & $\begin{array}{l}-0.63 \\
-0.66\end{array}$ & $\begin{array}{c}9.9 \\
110\end{array}$ & $\begin{array}{c}0-9.2 \\
698-805\end{array}$ & $\begin{array}{l}4 \\
4\end{array}$ & hotspot1 & $\begin{array}{l}9.3 \\
5.8\end{array}$ \\
\hline tco.1 & TCO & 2 & I & 81.9 & 3.10 & -0.84 & 10.6 & $80.5-85.0$ & 197 & $\begin{array}{c}\text { near to } \\
\text { hotspot } 20\end{array}$ & 467.7 \\
\hline
\end{tabular}

Note: ${ }^{a}$ CI: confidence intervals of QTLs. ${ }^{b}$ No. of genes: the number of genes in the confidence intervals of QTLs. ${ }^{c}$ Association with hotspots: the association of the physical location of QTLs and crossover hotspots.

For crossover variation of each chromosome, thirteen COchr-QTLs were distributed on six chromosomes, and both cis and trans factors contributed to local crossover variation. Of the thirteen COchr-QTLs, five were cis-prone QTLs, and eight were trans-QTLs (Table 1). An average of $20.1 \%$ of crossover number variation could be explained by cis-prone QTLs, which was higher than an average of $9.8 \%$ of trans-QTLs $(p=0.002)$. Of the cis-prone QTLs, the two adjacent loci cochr4.1 and cochr4.2 could explain up to $53.2 \%$ of the crossover variation of chr4. Additionally, the two QTLs were identified with high statistical power. The QTL cochr7.1 also contributed to more than $20 \%$ of the phenotypic variation. With CI of $2.5 \mathrm{cM}$, the physical length of cochr7.1 $(836.7 \mathrm{~Kb})$ covered nearly $24 \%$ of chr7. It was mainly due to the low recombination rate $(7.1 \mathrm{cM} / \mathrm{Mb})$ on chr7 [15]. For mapped trans-QTLs, most were distributed on chr1 and chr2. On chr1, four trans-QTLs were identified underlying COchr2 and COchr8. On chr2, three trans-QTLs for crossover variation of chr3 were clustered. Moreover, cochr3.2 was co-located with hco.2, while cochr3.3 was co-located with tco.1. These results indicated the presence of a QTL hotspot on chr2 (ranged from 69.8 to $87.6 \mathrm{cM}$ ), that regulates local and global crossover variations. Interestingly, this QTL hotspot underlying crossover variations was overlapped with the meta-QTL pq2.1 responsible for fruiting body-related traits [15]. In plants, such as maize, the significant associations between recombination events and variation in gene expression and agronomic traits were also identified [43]. Our previous studies have identified 246 phenotype-specific expressed genes underlying fruiting body-related traits [15]. Here, we analyzed the correlation of TCO and HCO with the expression of the 246 genes, and identified 47 significant crossover association signals in 37 genes (Supplementary Table S4). We also analyzed the correlation between TCO and $\mathrm{HCO}$ with the surveyed fruiting body-related traits of the $\mathrm{F}_{1}$ haploid progenies. No significant correlation was observed among crossovers and fruiting bodyrelated traits (data not shown). This might be due to the fact that only a small part of variation both for crossovers and fruiting body-related traits could be explained by this QTL hotspot. 


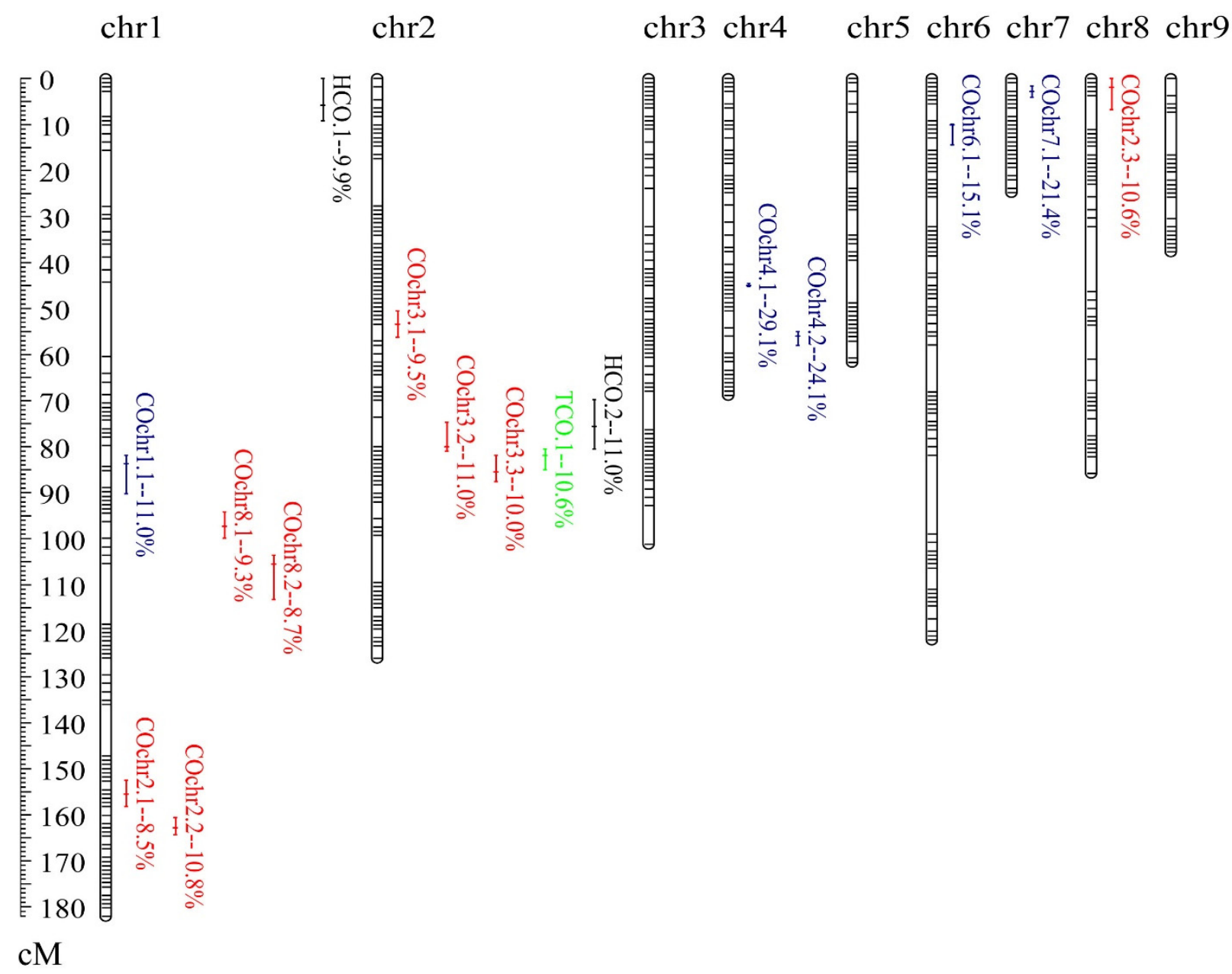

Figure 2. Distribution of QTLs for crossover variation in L. edodes. This Lemap2.0 was generated previously [15]. Sixteen QTLs for crossovers were shown on the right side of the chromosomes. The LOD-1 confidence interval was indicated by the length of the QTL bar, and the position of the LOD peak was represented by the short line in the middle of the QTL bar. The percentage value is represented the $\mathrm{R}^{2}$ value (percentage of explained phenotypic variation). The cis-prone QTLs are marked in blue, while trans-QTLs are in red. QTLs for HCO and TCO are marked in black and green, respectively.

The confidence intervals for the sixteen mapped crossover-QTLs ranged from 2.0 to $836.5 \mathrm{~kb}$, and contained a few to hundreds of genes (Table 1, Supplementary Table S5). Some well-known recombination-related genes were also found in the CIs of the QTLs. For instance, the Rad51 gene family is evolutionarily conserved in eukaryotes, and plays a critical role in double-strand break repair, replication stress and meiosis [44]. The homologous gene of Rad51C was identified in cochr4.1 (Supplementary Table S5). Moreover, the gene for Rad51-associated protein Brh2 was found in the mapped QTL hotspot underlying crossover variations (cochr3.3 and tco.1) (Supplementary Table S5). Brh2 works hand-in-hand with Rad51 to promote the repair of DNA by homologous recombination [45]. The HD1 gene encoding homeodomain protein was also included in this QTL hotspot (cochr3.2 and hco.2). Thereby, the co-localizations of mating-type genes, crossover-QTLs and crossover hotspots, may contribute to the mechanism whereby crossover frequencies around MAT-A loci were enhanced in L. edodes.

\section{Conclusions}

In summary, we elucidated the dynamics of intragenic crossover in $F_{1}$ haploid progenies of L. edodes. The 65 crossover hotspots and genetic architecture of crossover variation were characterized here. Enhanced crossovers adjacent to mating-type loci in L. edodes were also observed. QTL mapping revealed both cis- and trans-loci contributing to the nonuni- 
formity of crossover along chromosomes. A QTL hotspot on chr2 regulating local and global crossover variation and crossover hotspot was identified. The findings of this study advanced our understandings for the conservation and diversity of meiotic recombination in mushroom-forming fungi.

Supplementary Materials: The following are available online at https:/ /www.mdpi.com/article/10 .3390/jof7121076/s1, Figure S1: Variation of crossovers among the 110 SSIs. Figure S2: Significant differences of TCO and HCO among the SSIs with different MAT-A loci. Table S1: Crossovers in the 110 single spore isolates. Table S2: Table S2. Crossovers and lengths of nine chromosomes in L. edodes. Table S3: Distribution of crossover hotspot regions in L. edodes genome. Table S4: Associations of corssovers and genes regulating fruiting body-related traits. Table S5: Genes in confidence interval of QTLs for crossover variation in L.edodes. Supplementary File S1: findCrossover.py.

Author Contributions: Conceptualization, W.G. and Y.X.; Visualization, N.S. and L.Z.; Writing -Original Draft, W.G. and N.S.; Writing—Review and Editing, Y.B. and Y.X. All authors have read and agreed to the published version of the manuscript.

Funding: This work was funded by the Fundamental Research Funds for the Central Universities (Program No. 2662018PY097), and the National Natural Science Foundation of China (Grant No. 32070015).

Institutional Review Board Statement: Not applicable.

Informed Consent Statement: Not applicable.

Data Availability Statement: Data of this study are included in the article or Supplementary Materials.

Conflicts of Interest: The authors declare no conflict of interest.

\section{References}

1. Peñalba, J.V.; Wolf, J.B.W. From molecules to populations: Appreciating and estimating recombination rate variation. Nat. Rev. Genet. 2020, 21, 476-492. [CrossRef]

2. Lambing, C.; Tock, A.J.; Topp, S.D.; Choi, K.; Kuo, P.C.; Zhao, X.; Osman, K.; Higgins, J.D.; Franklin, F.C.H.; Henderson, I.R. Interacting Genomic Landscapes of REC8-Cohesin, Chromatin, and Meiotic Recombination in Arabidopsis. Plant Cell 2020, 32, 1218-1239. [CrossRef] [PubMed]

3. Kianian, P.M.A.; Wang, M.; Simons, K.; Ghavami, F.; He, Y.; Dukowic-Schulze, S.; Sundararajan, A.; Sun, Q.; Pillardy, J.; Mudge, J.; et al. High-resolution crossover mapping reveals similarities and differences of male and female recombination in maize. Nat. Commun. 2018, 9, 1-10. [CrossRef]

4. Mieulet, D.; Aubert, G.; Bres, C.; Klein, A.; Droc, G.; Vieille, E.; Rond-Coissieux, C.; Sanchez, M.; Dalmais, M.; Mauxion, J.-P.; et al. Unleashing meiotic crossovers in crops. Nat. Plants 2018, 4, 1010-1016. [CrossRef]

5. Choi, K.; Henderson, I. Meiotic recombination hotspots-A comparative view. Plant J. 2015, 83, 52-61. [CrossRef]

6. Wang, Y.; Copenhaver, G.P. Meiotic Recombination: Mixing It Up in Plants. Annu. Rev. Plant Biol. 2018, 69, 577-609. [CrossRef] [PubMed]

7. Gray, S.; Cohen, P.E. Control of Meiotic Crossovers: From Double-Strand Break Formation to Designation. Annu. Rev. Genet. 2016, 50, 175-210. [CrossRef]

8. Li, X.; Li, L.; Yan, J. Dissecting meiotic recombination based on tetrad analysis by single-microspore sequencing in maize. Nat. Commun. 2015, 6, 6648. [CrossRef]

9. Pan, Q.; Deng, M.; Yan, J.; Li, L. Complexity of genetic mechanisms conferring nonuniformity of recombination in maize. Sci. Rep. 2017, 7, 1-11. [CrossRef] [PubMed]

10. Dreissig, S.; Maurer, A.; Sharma, R.; Milne, L.; Flavell, A.J.; Schmutzer, T.; Pillen, K. Natural variation in meiotic recombination rate shapes introgression patterns in intraspecific hybrids between wild and domesticated barley. New Phytol. 2020, 228, 1852-1863. [CrossRef]

11. Seplyarskiy, V.B.; Logacheva, M.D.; Penin, A.A.; Baranova, M.A.; Leushkin, E.V.; Demidenko, N.V.; Klepikova, A.V.; Kondrashov, F.A.; Kondrashov, A.S.; James, T.Y. Crossing-Over in a Hypervariable Species Preferentially Occurs in Regions of High Local Similarity. Mol. Biol. Evol. 2014, 31, 3016-3025. [CrossRef]

12. Sonnenberg, A.S.; Gao, W.; Lavrijssen, B.; Hendrickx, P.; Sedaghat-Tellgerd, N.; Foulongne-Oriol, M.; Kong, W.-S.; Schijlen, E.G.; Baars, J.; Visser, R.G. A detailed analysis of the recombination landscape of the button mushroom Agaricus bisporus var. bisporus. Fungal Genet. Biol. 2016, 93, 35-45. [CrossRef] [PubMed]

13. Sheng, K.; Wang, C.; Chen, B.; Kang, M.; Wang, M.; Liu, K.; Wang, M. Recent advances in polysaccharides from Lentinus edodes (Berk.): Isolation, structures and bioactivities. Food Chem. 2021, 358, 129883. [CrossRef] [PubMed] 
14. Royse, D.J.; Baars, J.; Tan, Q. Current Overview of Mushroom Production in the World. In Edible and Medicinal Mushrooms; Wiley-Blackwell: Oxford, UK, 2017; pp. 5-13.

15. Zhang, L.; Gong, W.; Li, C.; Shen, N.; Gui, Y.; Bian, Y.; Kwan, H.S.; Cheung, M.K.; Xiao, Y. RNA-Seq-based high-resolution linkage map reveals the genetic architecture of fruiting body development in shiitake mushroom, Lentinula edodes. Comput. Struct. Biotechnol. J. 2021, 19, 1641-1653. [CrossRef] [PubMed]

16. Gong, W.-B.; Liu, W.; Lu, Y.-Y.; Bian, Y.-B.; Zhou, Y.; Kwan, H.S.; Cheung, M.K.; Xiao, Y. Constructing a new integrated genetic linkage map and mapping quantitative trait loci for vegetative mycelium growth rate in Lentinula edodes. Fungal Biol. 2014, 118, 295-308. [CrossRef]

17. Gong, W.-B.; Li, L.; Zhou, Y.; Bian, Y.-B.; Kwan, H.-S.; Cheung, M.-K.; Xiao, Y. Genetic dissection of fruiting body-related traits using quantitative trait loci mapping in Lentinula edodes. Appl. Microbiol. Biotechnol. 2016, 100, 5437-5452. [CrossRef]

18. Jordan, K.W.; Wang, S.; He, F.; Chao, S.; Lun, Y.; Paux, E.; Sourdille, P.; Sherman, J.; Akhunova, A.; Blake, N.K.; et al. The genetic architecture of genome-wide recombination rate variation in allo-polyploid wheat revealed by nested association mapping. Plant J. 2018, 95, 1039-1054. [CrossRef]

19. Gong, W.; Song, X.; Xie, C.; Zhou, Y.; Zhu, Z.; Xu, C.; Peng, Y. Landscape of meiotic crossovers in Hericium erinaceus. Microbiol. Res. 2021, 245, 126692. [CrossRef]

20. Si, W.; Yuan, Y.; Huang, J.; Zhang, X.; Zhang, Y.; Zhang, Y.; Tian, D.; Wang, C.; Yang, Y.; Yang, S. Widely distributed hot and cold spots in meiotic recombination as shown by the sequencing of rice F2 plants. New Phytol. 2015, 206, 1491-1502. [CrossRef]

21. Davis, T.M.; Yang, Y.; Mahoney, L.L.; Frailey, D.C. A pentaploid-based linkage map of the ancestral octoploid strawberry Fragaria virginiana reveals instances of sporadic hyper-recombination. Hortic. Res. 2020, 7, 1-15. [CrossRef]

22. Heinz, S.; Benner, C.; Spann, N.; Bertolino, E.; Lin, Y.; Laslo, P.; Cheng, J.X.; Murre, C.; Singh, H.; Glass, C.K. Simple Combinations of Lineage-Determining Transcription Factors Prime cis-Regulatory Elements Required for Macrophage and B Cell Identities. Mol. Cell 2010, 38, 576-589. [CrossRef]

23. Wang, S.; Bastern, C.J.; Zeng, Z.B. Windows QTL Cartographer 2; Department of Statistics, North Carolina State University: Raleigh, NC, USA, 2007; Available online: https:/ / brcwebportal.cos.ncsu.edu/qtlcart/WQTLCart.htm (accessed on 28 May 2021 ).

24. Lu, S.; Zong, C.; Fan, W.; Yang, M.; Li, J.; Chapman, A.R.; Zhu, P.; Hu, X.; Xu, L.; Yan, L.; et al. Probing Meiotic Recombination and Aneuploidy of Single Sperm Cells by Whole-Genome Sequencing. Science 2012, 338, 1627-1630. [CrossRef] [PubMed]

25. Ludlow, C.L.; Scott, A.C.; Cromie, G.A.; Jeffery, E.W.; Sirr, A.; May, P.; Lin, J.; Gilbert, T.L.; Hays, M.; Dudley, A.M. Highthroughput tetrad analysis. Nat. Chem. Biol. 2013, 10, 671-675. [CrossRef]

26. Wijnker, E.; James, G.V.; Ding, J.; Becker, F.; Klasen, J.R.; Rawat, V.; Rowan, B.; De Jong, D.F.; De Snoo, C.B.; Zapata, L.; et al. The genomic landscape of meiotic crossovers and gene conversions in Arabidopsis thaliana. eLife 2013, 2, e01426. [CrossRef] [PubMed]

27. Hunter, N. Meiotic Recombination: The Essence of Heredity. Cold Spring Harb. Perspect. Biol. 2015, 7, a016618. [CrossRef]

28. Croll, D.; Lendenmann, M.H.; Stewart, E.L.; McDonald, B. The Impact of Recombination Hotspots on Genome Evolution of a Fungal Plant Pathogen. Genetics 2015, 201, 1213-1228. [CrossRef]

29. Pérez, G.; Lopez-Moya, F.; Chuina, E.; Ibañez-Vea, M.; Garde, E.; López-Llorca, L.V.; Pisabarro, A.G.; Ramírez, L. Strain Degeneration in Pleurotus ostreatus: A Genotype Dependent Oxidative Stress Process Which Triggers Oxidative Stress, Cellular Detoxifying and Cell Wall Reshaping Genes. J. Fungi 2021, 7, 862. [CrossRef] [PubMed]

30. Pan, J.; Sasaki, M.; Kniewel, R.; Murakami, H.; Blitzblau, H.G.; Tischfield, S.E.; Zhu, X.; Neale, M.J.; Jasin, M.; Socci, N.D.; et al. A Hierarchical Combination of Factors Shapes the Genome-wide Topography of Yeast Meiotic Recombination Initiation. Cell 2011, 144, 719-731. [CrossRef]

31. Hou, Y.; Fan, W.; Yan, L.; Li, R.; Lian, Y.; Huang, J.; Li, J.; Xu, L.; Tang, F.; Xie, X.S.; et al. Genome Analyses of Single Human Oocytes. Cell 2013, 155, 1492-1506. [CrossRef]

32. Marand, A.P.; Zhao, H.; Zhang, W.; Zeng, Z.; Fang, C.; Jiang, J. Historical Meiotic Crossover Hotspots Fueled Patterns of Evolutionary Divergence in Rice. Plant Cell 2019, 31, 645-662. [CrossRef]

33. Foulongne-Oriol, M.; Taskent, O.; Kües, U.; Sonnenberg, A.; van Peer, A.; Giraud, T. Mating-Type Locus Organization and Mating-Type Chromosome Differentiation in the Bipolar Edible Button Mushroom Agaricus bisporus. Genes 2021, $12,1079$. [CrossRef]

34. Andrews, B.J.; Moore, L.A. Interaction of the yeast Swi4 and Swi6 cell cycle regulatory proteins in vitro. Proc. Natl. Acad. Sci. USA 1992, 89, 11852-11856. [CrossRef] [PubMed]

35. Giraut, L.; Falque, M.; Drouaud, J.; Pereira, L.; Martin, O.; Mézard, C. Genome-Wide Crossover Distribution in Arabidopsis thaliana Meiosis Reveals Sex-Specific Patterns along Chromosomes. PLoS Genet. 2011, 7, e1002354. [CrossRef]

36. Kong, A.; Thorleifsson, G.; Gudbjartsson, D.; Masson, G.; Sigurdsson, A.; Jonasdottir, A.; Walters, G.B.; Jonasdottir, A.; Gylfason, A.; Kristinsson, K.T.; et al. Fine-scale recombination rate differences between sexes, populations and individuals. Nat. Cell Biol. 2010, 467, 1099-1103. [CrossRef] [PubMed]

37. Luo, C.; Li, X.; Zhang, Q.; Yan, J. Single gametophyte sequencing reveals that crossover events differ between sexes in maize. Nat. Commun. 2019, 10, 1-8. [CrossRef] [PubMed]

38. Sun, S.; Heitman, J. 1 Running Hot and Cold: Recombination Around and Within Mating-Type Loci of Fungi and Other Eukaryotes. In Environmental and Microbial Relationships; Springer: Singapore, 2016; pp. 3-13. 
39. Larraya, L.M.; Pérez, G.; Iribarren, I.; Blanco, J.A.; Alfonso, M.; Pisabarro, A.G.; Ramírez, L. Relationship between Monokaryotic Growth Rate and Mating Type in the Edible Basidiomycete Pleurotus ostreatus. Appl. Environ. Microbiol. 2001, 67, 3385-3390. [CrossRef]

40. Idnurm, A.; Hood, M.E.; Johannesson, H.; Giraud, T. Contrasted patterns in mating-type chromosomes in fungi: Hotspots versus coldspots of recombination. Fungal Biol. Rev. 2015, 29, 220-229. [CrossRef]

41. Wang, R.; Payseur, B.A. Genetics of Genome-Wide Recombination Rate Evolution in Mice from an Isolated Island. Genetics 2017, 206, 1841-1852. [CrossRef]

42. Esch, E.; Szymaniak, J.M.; Yates, H.; Pawlowski, W.P.; Buckler, E. Using Crossover Breakpoints in Recombinant Inbred Lines to Identify Quantitative Trait Loci Controlling the Global Recombination Frequency. Genetics 2007, 177, 1851-1858. [CrossRef] [PubMed]

43. Pan, Q.; Li, L.; Yang, X.; Tong, H.; Xu, S.; Li, Z.; Li, W.; Muehlbauer, G.J.; Li, J.; Yan, J. Genome-wide recombination dynamics are associated with phenotypic variation in maize. New Phytol. 2016, 210, 1083-1094. [CrossRef]

44. Bonilla, B.; Hengel, S.R.; Grundy, M.K.; Bernstein, K.A. RAD51 Gene Family Structure and Function. Annu. Rev. Genet. 2020, 54, 25-46. [CrossRef] [PubMed]

45. Mazloum, N.; Zhou, Q.; Holloman, W.K. D-loop formation by Brh2 protein of Ustilago maydis. Proc. Natl. Acad. Sci. USA 2008, 105, 524-529. [CrossRef] [PubMed] 